\title{
Hereditary multiple exostoses: report of a kindred
}

\author{
STANLEY L GORDON, * JAMES R BUCHANAN, * AND ROGER L LADDA † \\ From * the Division of Orthopedic Surgery, Department of Surgery, and †the Division of Genetics, \\ Department of Pediatrics, The Pennsylvania State University College of Medicine, Hershey, \\ Pennsylvania, USA
}

SUMMARY In a large family with 37 members with multiple exostoses, only one person has developed sarcomatous degeneration of a lesion. Our review of published reports revealed great variation in the incidence of malignancy in multiple exostoses (10 to $25 \%$ ). Most studies had sampling errors leading to the apparent overstatement of risk. In large pedigrees with essentially complete ascertainment of $\bar{\rho}^{\infty}$ affected subjects, the risk of malignancy is nearer $3 \%$ or less. This lower risk for malignancy may be more appropriate in counselling affected subjects.

Hereditary multiple exostoses is an autosomal dominant trait characterised by numerous cartilage capped lesions located in areas of actively growing bone. ${ }^{1-3}$ Although exostoses may cause complications through the compression of local tissues, the major threat to life derives from the malignant degeneration of the lesions. Earlier studies of families with multiple exostoses ${ }^{4-9}$ reported on the wide variation in the severity of the trait and the risk of malignant transformation. We have recently had the opportunity to follow and treat an extended family of Pennsylvania German extraction. Because these families are large and stable, we were able to trace five generations and identify 37 cases of multiple exostoses out of 104 family members (table). This family study provides additional information on the incidence of sarcomatous degeneration in multiple exostoses which may be useful in the counselling and care of relatives at risk.

\section{Family studies}

With the assistance of one member of the family, questionnaires were sent to all other living members, seeking information on themselves, their parents, grandparents, children, and grandchildren. Specific data were obtained on names, dates of birth, dates and causes of death, and degree of involvement. Subjects were asked to estimate bony involvement as mild (three or fewer lesions), severe (multiple lesions usually with significant deformity in one or more extremities), or moderate. Since the family was aware of the relationship between bone malignancies and osteochondromatoses and was sensitised to the sinister implications of enlarging lesions, precise information was available for detection of bony malignancies. Every living family member responded

TABLE Affected subjects

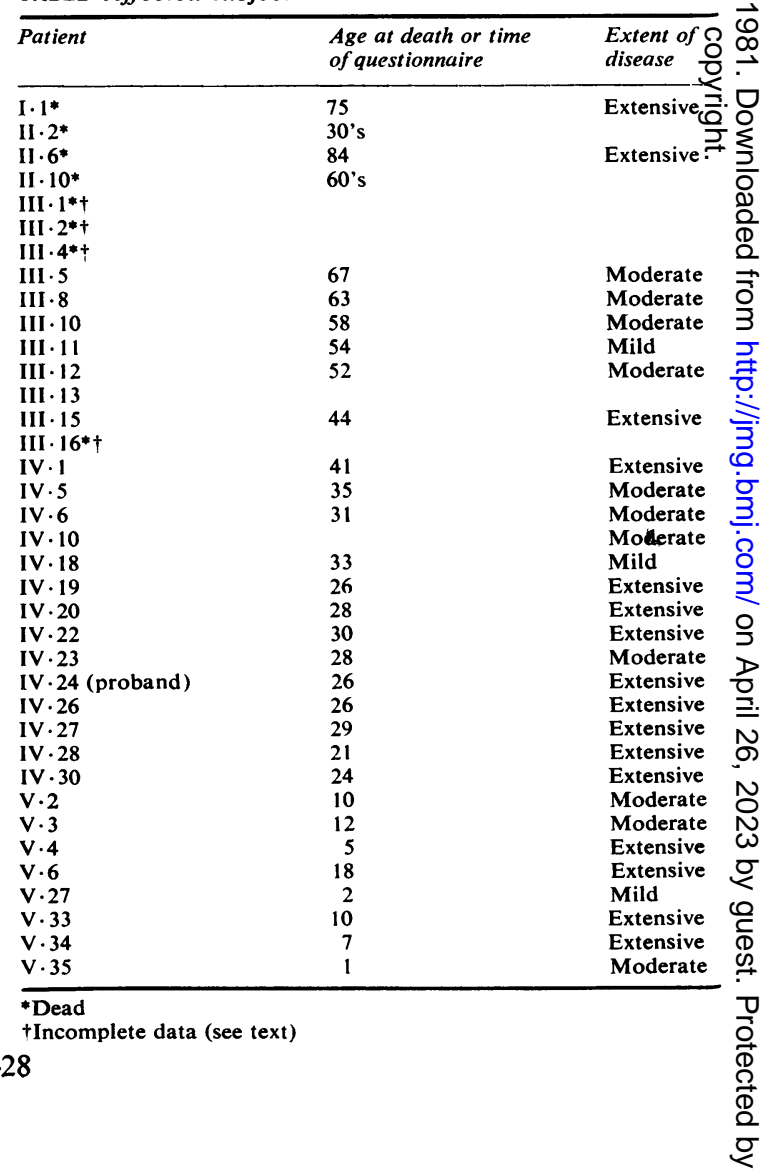




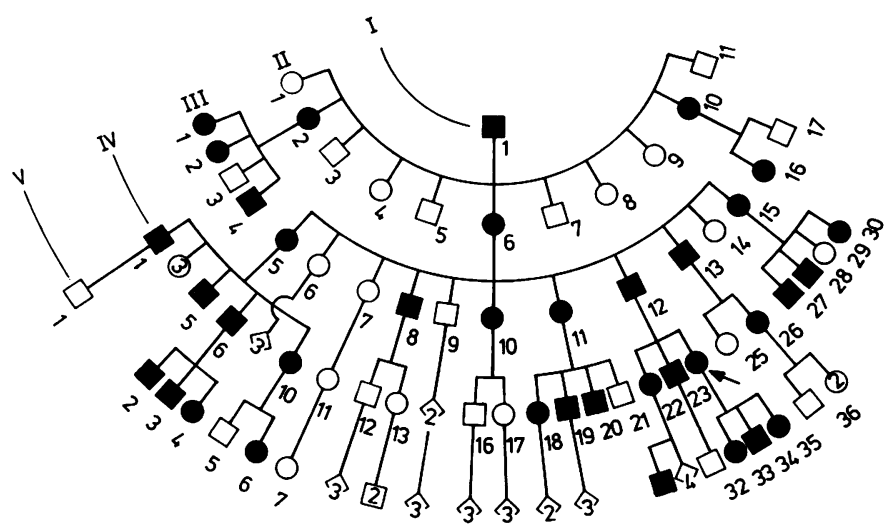

FIG 1 Pedigree of five generations. Not included are the brothers and sisters, parents, wife, or wives' brothers and sisters for generation I, none of whom had evidence of the disease. to the questionnaire. Long, personally written letters were analysed and compared, permitting tracing of every member of the pedigree. Further information was obtained from family reunions and functions so that descriptions of dead relatives and ancestors could be compared. Because of intimate family ties, sufficient information about previous generations could be obtained to include accurate physical descriptions.

The founding couple of the family immigrated to Central Pennsylvania late in the 18th century. The first instance of multiple exostoses appeared in a male born in $1854(\mathrm{I} \cdot 1$, fig 1$)$, who was described as having multiple lesions with short arms and angular deformity at the elbows. He had nine brothers and sisters, but neither they nor their parents were clinically afflicted with multiple exostoses. There was no history of the disease in his wife's relatives. Three of his nine children were afflicted with multiple osteochondromatoses and passed the disease to the next generation. Exact birth dates and death dates could not be established for five childless members from the second generation. Four of the five had the disease, but none of them developed bony malignancies during their lifetime. A total of 104 members in five generations was investigated. Of these, 37 subjects had multiple exostoses (17 males and 20 females). Most members of the family lived to extended ages in apparent good health without known disability. Only one person had sarcomatous bone degeneration. There was no family history of other congenital malformations.

\section{Case report}

Subject IV.27, a product of a normal gestation, labour, and delivery, was noted to have extensive multiple exostoses shortly after birth. During his childhood and adolescence, osteochondromata were removed from his pelvis and humerus. In April 1971, at the age of 23 , an exostosis was removed from his left scapula (fig 2) and this showed malignant changes. Histopathological examination showed a cartilaginous tumour with increased cellularity, pleomorphism, chromatin clumping, and multinucleate cells in numerous lacunae (fig 3 ). Accordingly, a radical scapulectomy was performed for what appeared to be a low grade chondrosarcoma. In December 1971, a local recurrence in the posterior chest was excised and subsequently skin grafted. No further recurrence has occurred in the past 8 years.

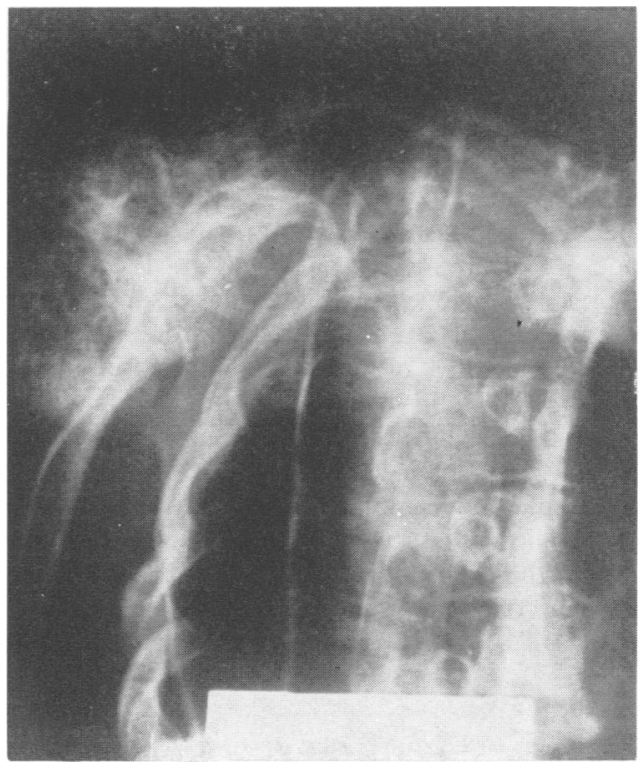

FIG $2 X$-ray of chondrosarcoma of scapula in $I V \cdot 27$. 


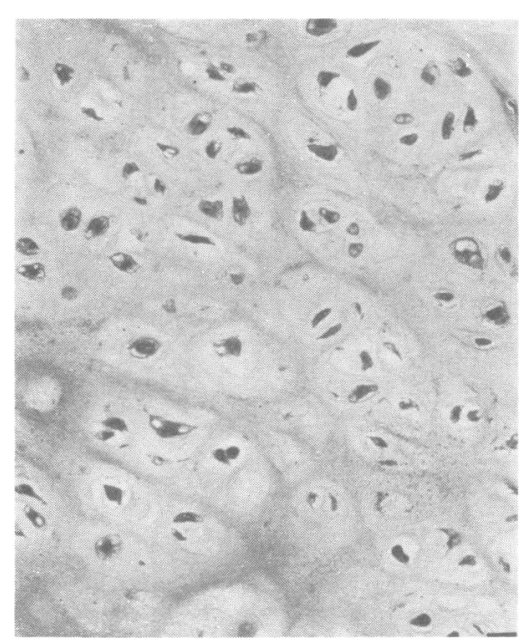

FIG 3 Slide of chondrosarcoma showing abnormal pleomorphic cells and multicellular lacunae.

\section{Discussion}

Physicians are frequently asked about the exact risk of bony malignancy by anxious subjects with hereditary multiple exostoses. A careful family history may be the most important factor for predicting the probable risk of malignant degeneration. The reported incidence of malignant degeneration of the exostoses varies greatly, ranging from 3 to $25 \%$. Chondrosarcoma appeared in three of the 28 patients studied by Jaffe ${ }^{10}$ at the Hospital for Joint Disease. However, only those family members evaluated at the hospital were included in this estimate and thus ascertainment was not complete. Based on a review of 272 cases of multiple exostoses treated at the Mayo Clinic, Dahlin ${ }^{1}$ estimated the incidence of chondrosarcoma to be about $10 \%$. None of the index patients in the series of Solomon ${ }^{6} 711$ developed secondary sarcoma. Only one case of sarcomatous degeneration was found in the large family of Vanzant ${ }^{9}$ which included 36 affected subjects in five generations. Similarly, in our large pedigree, only one documented chondrosarcoma was detected in about 1000 risk years of follow-up. It is possible that the incidences of Jaffe ${ }^{10}$ and Dahlin ${ }^{1}$ are spuriously high since they did not evaluate the entire population at risk. It appears that the risk of malignant degeneration in affected subjects is in fact low, albeit greater than the risk tod the general population. The relatively low incidenced of malignancy in some families and apparent high: incidence in others may reflect allelic forms ofos? multiple exostoses, chance occurrence, environmentato effects, epistasis, or some combination of theseo factors.

Although most cases of sarcomatous degeneration $\frac{\Phi}{\alpha}$ occur in adults, it has been reported in subjects aso young as 10 years of age. ${ }^{4} \mathrm{Knight}^{5}$ reported bony ${ }^{\text {s }}$ sarcomatous changes in three brothers with the disease. Once the diagnosis is established, it is only $\overrightarrow{\vec{H}}$ prudent to alert the family of the potential risk of malignancy. An enlarging lesion at any age needs immediate attention. In a large family with many. affected members, the risk of malignancy may beop estimated from previous familial occurrences. Foros those subjects who represent new mutations (about $40 \%$ of the newly detected affected subjects), the ${ }^{\infty}$ risk of malignancy is probably about $3 \%$ and perhaps even less.

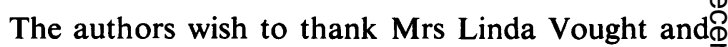
Dr Paul Sweterlitsch for their assistance in preparing $\overline{3}$ this manuscript.

\section{References}

1 Dahlin DC. Bone tumors, Springfield, Illinois: Thom 1957.

2 Huvos AG. Bone tumors, diagnoses, and treatment $\underset{a n d}{\overrightarrow{7}}$ prognosis. Philadelphia: Saunders, 1979.

3 Wynne-Davies R. Heritable disorders in orthopaedico practice. Oxford: Blackwell Scientific Publications, 1973. श

4 Bennett GE, Berkheimer GA. Malignant degeneration in a case of multiple benign exostoses. Surgery $1941 ; 10: 781-92 . \overline{\mathrm{o}}$

5 Knight JDS. Sarcomatous change in three brothers with diaphyseal aclasis. $B r$ Med $J 1960$;i:1013-4.

6 Solomon L. Hereditary multiple exostosis. J Bone Join हि Surg (Br) 1963;45:292-304. 7 Solomon L. Hereditary multiple exostosis. Am J Hum

${ }^{8}$ Stocks P, Barrington A. Hereditary disorders of boneo development. In: The treasury of human inheritance. VoB. III. London: Cambridge University Press, 1925.

9 Vanzant BT, Vanzant FR. Hereditary deforming chondrodysplasia. JAMA 1942;119:786-90.

10 Jaffe HL. Hereditary multiple exostosis. Arch Patho윽 $1943 ; 36: 335-56$.

11 Solomon L. Chondrosarcoma in hereditary multiple exostosis. S Afr Med J 1974;48:671-4.

Requests for reprints to Dr S L Gordon, Division of Orthopedic Surgery, Milton S Hershey Medicalo Center, Hershey, Pennsylvania 17033, USA. 